\title{
Religionsverfassungsrecht und säkulare Gesellschaft - Kritische Anmerkungen zur Abteilung Öffentliches Recht des Juristentages 2010
}

Johannes Rux"

Unter dem Thema „Neue Religionskonflikte und staatliche Neutralität - Erfordern weltanschauliche und religiöse Entwicklungen Antworten des Staates? “ diskutierte die Abteilung für Öffentliches Recht des 68. Deutschen Juristentages in Berlin Ende September über einen echten Dauerbrenner der Staatsrechtslehre - und zugleich über ein hochaktuelles Thema, da die gegenwärtige Diskussion über Integrationsfähigkeit und Integrationsbereitschaft von Einwanderern vor allem auf diejenigen Menschen zielt, die in den letzten Jahren und Jahrzehnten aus islamisch geprägten Kulturen nach Deutschland eingewandert sind. Nachdem sich immer mehr Menschen für eine Einschränkung der Religionsfreiheit zu Lasten der Muslime aussprechen ${ }^{1}$ und in einigen Nachbarstaaten Deutschlands solche Einschränkungen in den Parlamenten beraten oder bereits - wie etwa das französische Burka-Verbot - den Weg in die Gesetzblätter gefunden haben, versprach das Thema spannende Diskussionen. ${ }^{2}$

Paul Kirchbof begann sein Referat mit der plakativen, aber eingängigen Forderung nach einem staatlichen Schutzschirm für die Religionsgemeinschaften. Es bedürfe religiöser Institutionen, um die kulturellen Grundlagen für das Zusammenleben in der Gesellschaft zu schaffen und zu erhalten. Nachdem die moderne Wissenschaft den Menschen aus dem Zentrum gerückt habe, sei dies wichtiger denn je: Angesichts der technischen Entwicklung komme es heute nämlich nicht mehr darauf an, was man kann, sondern vielmehr darauf, was man will. Um diese Entscheidung treffen zu können, brauche es jedoch eines Rahmens, den nach Kirchhofs Ansicht alleine die Religionsgemeinschaften schaffen können.

Kirchhof betonte dabei durchaus die Freiheit des Einzelnen, sich einen Glauben zu bilden und sich gegebenenfalls auch religiöser Überzeugungen zu enthalten. Der individuellen Glaubens- und Bekenntnisfreiheit komme im modernen Verfassungsstaat eine herausragende Rolle zu. Allerdings seien der Religionsunterricht an den Schulen und die religiöse Erziehung durch die Eltern essentielle Voraussetzung für die Mündigkeit des Einzelnen und notwendige Bedingung für den Fortbestand des Kultur-

* PD Dr. Johannes Rux ist Privatdozent für Öffentliches Recht an der Eberhard-Karls-Universität in Tübingen und Schriftleiter der RECHTSWISSENSCHAFT.

1 So zuletzt eine Studie der Friedrich-Ebert-Stiftung, deren Ergebnisse Anfang Oktober 2010 veröffentlicht wurden, vgl. http://www.faz.net/-01irl4 (abgerufen am 22.10.2010).

2 Wie aufgeheizt die Stimmung ist, zeigt etwa ein Gastbeitrag des renommierten Doyens der deutschen Völkerrechtswissenschaft Karl Doehring, der unter dem Titel „Niemand kann zwei Herren dienen“ aus Anlass des Juristentages in der FAZ „dem Islam“ per se die Vereinbarkeit mit dem Grundgesetz ab- und sich dementsprechend gegen die Einführung eines islamischen Religionsunterrichts aussprach, http:// www.faz.net/-01i57v (abgerufen am 22.10.2010). 
staates. Um diese These zu untermauern, verglich Kirchhof die religiöse Erziehung mit dem Musikunterricht: Wenn sich Eltern dazu entschließen, ihr Kind ein bestimmtes Instrument lernen zu lassen, sei dies keine endgültige Festlegung. Vielmehr würden die Eltern damit lediglich die Grundlage dafür schaffen, dass sich ihr Kind später frei entscheiden kann: Wer als Kind gelernt hat Geige zu spielen, mag als Erwachsener in der Tat Spaß am Schlagzeug finden oder ganz darauf verzichten, selbst Musik zu machen. Überträgt man dieses Bild auf die religiöse Erziehung, so würden Eltern, die ihr Kind im Sinne eines bestimmten Glaubens erziehen, ihm lediglich die allgemeine Bedeutung von Spiritualität und der Möglichkeit einer transzendentalen Begründung von Werten vermitteln und ihm im Übrigen die Freiheit belassen, sich später für einen anderen Glauben oder - Gott behüte - gegen jede religiöse Überzeugung zu entscheiden.

Christoph Möllers betonte in seinem Referat zunächst, dass sich Deutschland schon immer - zumindest seit der Reformation - durch eine große religiöse Pluralität ausgezeichnet habe. Noch in der Zeit der Weimarer Republik sei religiöse Vielfalt im Alltag eine Selbstverständlichkeit gewesen. Der irreführende Eindruck religiöser Homogenität und eines christlich geprägten deutschen Staates sei erst in den Jahren nach dem Zweiten Weltkrieg entstanden. Dieser Eindruck habe aber wiederum auch die Rechtsprechung insbesondere des Bundesverfassungsgerichts beeinflusst, das den Geltungsbereich der Religionsfreiheit in seinen frühen Entscheidungen über Gebühr ausgedehnt habe. Angesichts des offensichtlichen religiösen Pluralismus sei es durchaus zweifelhaft, ob eine Entscheidung, wie das bahnbrechende Lumpensammler-Urteil des Bundesverfassungsgerichts, ${ }^{3}$ mit dem die gesamte karitative Tätigkeit der christlichen Religionsgemeinschaften dem Schutz der Religionsfreiheit unterworfen wurde, heute noch so ergehen würde.

Nachdem die Religionsfreiheit heute deutlich restriktiver interpretiert werde als noch vor wenigen Jahren, erübrige sich ein Einschreiten des Gesetzgebers. Vielmehr reiche die Erkenntnis aus, dass nicht alles, was Religionsgemeinschaften tun, notwendigerweise Religionsausübung sein muss, und dass nicht alle Formen der Religionsausübungen notwendigerweise rechtmäßig sein müssen. Die aktuellen Probleme lassen sich nach Möllers Ansicht durchweg auf Grundlage der bestehenden Regelungen angemessen auflösen. Er sprach sich daher - wie später auch das Plenum - ausdrücklich für die Beibehaltung der „offenen Neutralität“ des Staates aus, der allen Religionsgemeinschaften gleichermaßen Entfaltungsmöglichkeiten geben müsse - wobei Möllers an zahlreichen Beispielen vom Kopftuchverbot im öffentlichen Dienst über das Aussageverweigerungsrecht zugunsten Geistlicher bis hin zum Religionsunterricht klar machte, dass dieser Grundsatz keineswegs immer beachtet wird.

3 BVerfGE 24, 236 - Aktion Rumpelkammer. 
Letzten Endes stellte aber auch Möllers nicht in Frage, dass es in erster Linie Aufgabe der - wie auch immer organisierten - Religionsgemeinschaften sein soll, durch die Vermittlung bestimmter Wertvorstellungen die Basis für die freie Entfaltung des einzelnen Menschen zu schaffen.

Wie eigentlich fast zu erwarten war, erwies sich der Deutsche Juristentag (auch) hier als außerordentlich konservativ. Die geltenden Bestimmungen des Staatsorganisationsrechts über das Verhältnis von Staat und Religionsgemeinschaften wurden durchgängig für ausreichend befunden, um Konflikte einer angemessenen Lösung zuzuführen. Selbst die vergleichsweise moderaten Reformvorschläge des Gutachters Christian Waldhoff zur Einführung eines besonderen Status zugunsten religiöser Vereine, die sich (noch) nicht als öffentlich-rechtliche Körperschaften konstituieren können oder wollen, wurden mit großer Mehrheit abgelehnt. Im Grunde scheinen die Verhandlungen der öffentlich-rechtlichen Abteilung des Juristentages daher allenfalls insofern bemerkenswert, als der Gesetzgeber gerade nicht gefordert wurde.

Tatsächlich geben sowohl das Gutachten von Christian Waldhoff als auch die beiden Referate von Paul Kirchbof und Christoph Möllers und auch die anschließende Diskussion Anlass, sich noch einmal etwas genauer mit der Fragestellung auseinanderzusetzen: Angesichts der unbestreitbaren Tatsache, dass nicht nur die christlichen Kirchen, sondern alle Religionsgemeinschaften zunehmend ihre Bindungskraft einbüßen und sich ein immer größerer Teil der Bevölkerung zu gar keinem Glauben mehr bekennen mag, ${ }^{4}$ verwundert die Einigkeit, mit der insbesondere den Religionsgemeinschaften die Aufgabe zugewiesen wurde, eine Begründung für die Geltung der Grundwerte der Verfassung zuliefern. Dies gilt umso mehr, wenn man berücksichtigt, dass auch ein immer größerer Teil derjenigen Menschen, die formal einer Religionsgemeinschaft angehören, tatsächlich alles andere als fest im Glauben sind und wohl keiner solchen Gemeinschaft angehören würden, wenn sie nicht schon als Kind durch die Taufe oder ein anderes Ritual Mitglied geworden wären ${ }^{5}$ - dies gilt wohl-

4 Nach den Statistiken des Religionswissenschaftlichen Medien- und Informationsdienstes e. V. (REMID) ist die Zahl der Säkularen im Jahr 2009 größer gewesen als die Mitgliederzahl der katholischen Kirche, (vgl. http://www.remid.de/remid_info_zahlen.htm - abgerufen am 22.10.2010). Ganz ähnliche Zahlen veröffentlicht die Forschungsgruppe Weltanschauungen in Deutschland (http://fowid.de/fileadmin/datenarchiv/Religionszugehoerigkeit_Bevoelkerung_1970_2010.pdf - abgerufen am 22.10.2010).

5 Hier steht die Nagelprobe unmittelbar bevor, nachdem der Europäische Gerichtshof für Menschenrechte am 23. September 2010, also praktisch zeitgleich mit den abschließenden Beratungen des Juristentags die in Deutschland bisher weitgehend unangefochtenen Sonderregelungen des kirchlichen Arbeitsrechtes zumindest für diejenigen Beschäftigten aufgehoben hat, deren Tätigkeit nicht unmittelbar dem Verkündigungsauftrag der Kirchen dient (EGMR Beschlüsse vom 23.9.2010 425/03 - Obst und 16020/03 Schüth). Dies betrifft aber insbesondere die von kirchlichen Trägern betriebenen Sozialen Einrichtungen von der Kinderkrippe bis zum Altenheim, deren Beschäftigte bisher damit rechnen mussten, dass der Austritt aus der Kirche oder ein Verstoß gegen die kirchlichen Sexual- oder Ehevorschriften ein Kündigungsgrund sein könnte. 
gemerkt auch für den Islam. ${ }^{6}$ Angesichts dieses Befundes bleibt nämlich völlig offen, nach welchen Maßstäben diejenigen Menschen ihr Verhalten ausrichten (sollen), die nicht an eine gottgegebene Wertordnung glauben. Darf es der Staat wirklich hinnehmen, wenn ein erheblicher Teil seiner Bürger letztendlich orientierungslos bleibt?

Tatsächlich kann davon keine Rede sein. Spätestens seit Beginn der Aufklärung hat der Staat das Recht für sich in Anspruch genommen, seine Bürger zu erziehen. Dies gilt für die mit absoluter Autorität herrschenden Fürsten des 18. Jahrhunderts ebenso wie für den modernen demokratischen Rechtsstaat. Besonders deutlich wird der Erziehungsanspruch des Staates am Beispiel der allgemeinen Schulpflicht, die unter anderem eingeführt wurde, um Kindern und Jugendlichen bestimmte Werte und Tugenden zu vermitteln. Auch wenn sich diese Werte oft religiös begründen lassen, ist dies keineswegs immer der Fall. Vielmehr können (und konnten von Anfang an) die Ziele der Erziehung in der Schule durchaus im Widerspruch jedenfalls zu radikalen Auslegungen der verschiedenen heiligen Schriften stehen.

Der Erziehungsanspruch des Staates endet keineswegs mit der Schulpflicht und dem Eintritt der Volljährigkeit: Geht man - im Gegensatz zu einer bis heute weit verbreiteten Ansicht - davon aus, dass der freiheitliche Staat den Bürgern ihre Freiheit nicht gewähren kann, sondern diese Freiheit vielmehr als gegeben voraussetzt, dann eröffnet dies nicht nur eine ganz andere Perspektive auf die Grundrechte des Grundgesetzes, ${ }^{7}$ sondern macht deutlich, dass ein solcher Staat nur durch die Verbreitung derjenigen Werte, die der Verfassungsordnung zugrunde liegen, die Grundlage und den Fortbestand seiner eigenen Existenz absichern kann. Das Bundesverfassungsgericht geht daher in ständiger Rechtsprechung ${ }^{8}$ völlig zu Recht davon aus, dass die Grundrechte nicht nur Grundlage und Maßstab der staatlichen Entscheidungen sind, sondern Ausdruck einer „objektiven Wertordnung“ sind, die auch das Verhalten zwischen Privaten prägt und damit weit über den Bereich des Rechts hinaus wirkt. Um seinem in Art. 1 GG statuierten Auftrag zum Schutz der Grundrechte gerecht zu werden, muss der Staat konsequenterweise darauf hinwirken, dass die Bürger diese Grundrechte auch als Maßstab ihres eigenen Verhaltens anerkennen oder zumindest respektieren.

Es liegt auf der Hand, dass eine staatlich gesteuerte Erziehung in einem totalitären System zur Indoktrination - im Sinne der Vermittlung einer bestimmten Ideologie

6 Insofern ist zu beachten, dass es keine islamische Religionsgemeinschaft gibt. Wenn in Statistiken davon die Rede ist, dass in Deutschland mittlerweile über 4 Millionen Muslime leben, die größtenteils gläubig seien, dann beruhen diese Angaben auf repräsentativen Umfragen und der Selbstauskunft der Betroffenen: Wer sich selbst schon nicht als Muslim definiert, fällt damit aus der Statistik heraus, vgl. dazu etwa Bundesamt für Migration und Flüchtlinge/Deutsche Islamkonferenz (Hrsg.), Muslimisches Leben in Deutschland, Nürnberg 2009, S. $57 \mathrm{ff}$.

7 Diese erweisen sich aus dieser Perspektive nicht als Freiheitsgewährleistungen, sondern vielmehr als differenziertes System zur Legitimation von Freiheitsbeschränkungen.

8 Seit BVerfGE 7, 198 - Lüth. 
als geschlossenem Wertsystem - werden kann oder sogar werden muss. Das schließt jedoch keineswegs aus, dass der Staat selbst für diejenigen Grundwerte wirbt, die der Verfassungsordnung zugrunde liegen.

Die entscheidende Frage ist damit aber, wer über die „Grundwerte der Verfassung “ entscheiden darf: Folgt man den Thesen, die auf dem Juristentag zu hören waren, so stünde es dem Staat nicht zu, diese Werte zu definieren. Dahinter steht wohl die unausgesprochene Annahme, dass „Erziehungs-“ oder "Staatsziele“, die vom Gesetzgeber festgeschrieben wurden, zumindest theoretisch jederzeit wieder geändert werden können - und damit jedenfalls auf den ersten Blick willkürlich erscheinen.

Tatsächlich sind diejenigen Werte, die einer freiheitlichen Verfassungsordnung zugrunde liegen, keineswegs Ausdruck der Willkür des Gesetzgebers. Andernfalls ließe sich kaum erklären, wieso diese Grundwerte mit gewissen Nuancierungen fast universell gelten. Sie stellen in gewisser Weise ein Destillat der gemeinsamen Überzeugungen der Bürger dar, wobei es nicht darauf ankommt, ob diese Überzeugungen transzendental begründet sind - sich der einzelne Mensch also aufgrund eines göttlichen Gebotes zu einem bestimmten Verhalten verpflichtet fühlt - oder ob sie auf der mehr oder weniger rationalen, möglicherweise strikt utilitaristischen Erkenntnis des Einzelnen beruhen, dass sich die betreffenden Werte besonders gut als allgemeiner Verhaltensmaßstab eignen.

Das viel zitierte und allzu häufig irrigerweise normativ verstandene ${ }^{9}$ Diktum Wolfgang Böckenfördes, nach dem der freiheitliche, säkularisierte Staat von Voraussetzungen lebe, die er selbst nicht garantieren kann, darf daher nicht in dem Sinne verstanden werden, dass sich ein freiheitlicher Staat jeder Erziehungsbemühungen enthalten müsste. Nahezu das Gegenteil ist der Fall: Es gehört zum Wesen einer freiheitlichen Demokratie, dass die maßgeblichen Grundwerte durch den Gesetzgeber identifiziert werden. Er allein ist dazu berufen, die Erziehungs- und Staatsziele zu formulieren und den Rahmen für die Ausübung der Staatsgewalt festzulegen. Und es ist damit auch eine der wichtigsten Aufgaben des Staates, den Bürgern diejenigen Grundwerte zu vermitteln, die der Verfassungsordnung zugrunde liegen und damit einen ganz maßgeblichen Beitrag dazu zu leisten, dass die Bürger diese Werte für sich akzeptieren.

Selbstverständlich kann sich der Staat dazu der Hilfe von Religionsgemeinschaften und anderen Institutionen der Zivilgesellschaft bedienen. Tatsächlich wird er seinem selbst gesetzten Anspruch ohne diese Hilfe kaum gerecht werden können. Insbesondere kann es für die Verbreitung demokratischer und freiheitlicher Prinzipien hilfreich sein, wenn Religionsgemeinschaften ihren Gläubigen aufzeigen, dass diese

9 Schon aus dem Kontext des mit „Die Entstehung des Staates als Vorgang der Säkularisation“ betitelten Beitrags (E.-W. Böckenförde, Staat, Gesellschaft, Freiheit, Frankfurt 1976, S. 60) geht hervor, dass es Böckenförde um die Darstellung einer historischen Entwicklung ging. 
Prinzipien mit den jeweiligen Glaubensgeboten vereinbar sind oder sogar aus solchen Geboten abgeleitet werden können. Aus der Perspektive der Aufklärung stellt sich allerdings die Frage, ob es wirklich zu begrüßen ist, wenn ein Teil der Bürger die Grundwerte der Verfassung nur oder vor allem deshalb für sich akzeptiert, weil und insoweit diese Werte auch transzendental begründet oder als Ausdruck einer göttlichen Offenbarung verstanden werden können. ${ }^{10}$ Angesichts des zuvor dargestellten religionssoziologischen Befundes kann und darf sich der Staat jedoch insofern nicht mehr vollständig oder zumindest weitgehend auf die Religionsgemeinschaften verlassen. Dies gilt umso mehr, als sich neben den christlichen Großkirchen und anderen traditionellen Religionsgemeinschaften zahlreiche religiöse Gruppierungen entwickelt haben, die dem Bedürfnis Einzelner nach Spiritualität durch eine fundamentalistische Auslegung der verschiedenen heiligen Schriften Rechnung tragen - wobei dies keineswegs nur oder auch nur in erster Linie den Islam betrifft. ${ }^{11}$

Damit wird aber deutlich, dass die wohl größte Herausforderung für den Staat und die Gesetzgeber im Bund und den Ländern keineswegs darin besteht, Regeln für den Umgang mit dem Islam zu finden, da sich das Prinzip der offenen Neutralität in der Tat auch insofern bewähren wird. Vielmehr stellt sich die Frage, wie der Staat seiner ureigenen Aufgabe, die Grundwerte der Verfassung zu verbreiten, in Zukunft nachkommen kann.

Die bisherigen Antworten auf diese Frage können nicht befriedigen. Denn diese bestehen etwa schlicht darin, diejenigen Jugendlichen, die nicht am Religionsunterricht teilnehmen, zum Besuch eines wie auch immer ausgestalteten Ethik-Unterrichts zu verpflichten. Denn dieser Unterricht wird oft ${ }^{12}$ erst für solche Schüler angeboten, die bereits religionsmündig sind. Dabei müssten die Grundlagen sehr viel früher gelegt werden. Zudem erscheint es angesichts der zunehmenden religiösen Pluralität mehr als angemessen, wenn es der Staat als seine Aufgabe ansehen würde, allen Kindern und Jugendlichen die Vielfalt der Begründungsmöglichkeiten in einem für alle obligatorischen Ethikunterricht nahezubringen und damit zugleich die Gemeinsamkeiten dieser Begründungsansätze herauszuarbeiten - während im konfessionellen Religionsunterricht zwangsläufig die Unterschiede in den Vordergrund gestellt werden können und müssen.

Auch sonst stünde es dem Staat gut an, aus dem Grundsatz der offenen Neutralität nicht nur ein Gebot abzuleiten, alle Religionsgemeinschaften gleichermaßen zu för-

10 Denn damit wird zugleich die Geltung derjenigen Werte in Frage gestellt, die sich nicht ohne weiteres mit den Glaubenssätzen der jeweiligen Religionsgemeinschaft vereinbaren lassen.

11 Die bei weitem meisten Anträge auf eine Befreiung von der Schulpflicht werden etwa von Angehörigen radikal-christlicher Gruppierungen gestellt, die geltend machen, dass die vom Staat vorgegebenen (und zumindest grundsätzlich auch für private Ersatzschulen geltenden) Erziehungsziele mit den Geboten ihres Glaubens unvereinbar seien.

12 In immer mehr Ländern (etwa in Sachsen und Thüringen) gibt es allerdings auch schon in den Grundschulen entsprechende Angebote. 
dern - und dieses Gebot auch endlich in die Praxis umzusetzen. ${ }^{13}$ Vielmehr brauchen auch diejenigen Menschen, die keiner Religionsgemeinschaft angehören oder ihren Glauben verloren haben, Foren der Selbstvergewisserung. Es reicht eben nicht aus, wenn der Staat in pluralistisch besetzten Gremien vom Rundfunkrat bis zu Ethikkommissionen neben den üblichen Vertretern der christlichen Großkirchen auch einen Muslim beruft, sondern er muss auch säkulare Institutionen fördern, damit sich diese in politische und gesellschaftliche Prozesse einbringen und den Bürgern aufzeigen, dass es möglich ist, die Geltung der Grundwerte der Verfassung auch ohne Rückgriff auf Glaubensgebote zu begründen. Entscheidungsverfahren, die auf eine Einbeziehung der Zivilgesellschaft angelegt $\operatorname{sind},{ }^{14}$ müssen so umgestaltet werden, dass auch solche Interessen eingebracht werden können, die sich nicht ohne Weiteres organisieren lassen. ${ }^{15}$ Das alles ist wahrscheinlich noch sehr viel schwieriger und bedarf noch mehr legislative Kreativität, als einen - oder mehrere ${ }^{16}$ - Ansprechpartner zu finden, der die islamische Perspektive einbringt. Aber wer sonst, außer dem Staat, könnte diese Aufgabe erfüllen?

In den Beratungen des Juristentages wurde das grundlegende Dilemma der religionsverfassungsrechtlichen Diskussion deutlich, die sich vor allem dadurch auszeichnet, dass an dieser Diskussion fast durchweg nur solche Personen beteiligt sind, die selbst religiös gebunden und oft sogar institutionell mit einer Religionsgemeinschaft verbunden sind ${ }^{17}$ - und unter den wenigen Ausnahmen finden sich viele Vertreter eines radikalen Laizismus, der seinerseits schon fast wieder religiöse Züge trägt. Auch im rechtswissenschaftlichen Diskurs haben die Religionsgemeinschaften damit einen Einfluss, der deutlich größer ist als ihre gesellschaftliche Bedeutung. Vielleicht ist es an der Zeit, den Diskurs zu öffnen - dazu hätte der Juristentag durch eine andere Auswahl der Gutachter und Referenten und vielleicht auch durch eine etwas offenere Formulierung des Themas einen wichtigen Beitrag leisten können. Schade, dass er es nicht getan hat.

13 Bis heute haben die großen christlichen Kirchen eine deutlich privilegierte Stellung, die sich nicht nur daraus ergibt, dass das Staatskirchenrecht auf sie zugeschnitten ist. Vielmehr genießen sie aufgrund von Konkordaten aus vorkonstitutioneller Zeit Sonderrechte, die trotz des bereits in Art. 138 WRV formulierten Verfassungsauftrags immer noch nicht abgelöst sind.

14 Dies sind nicht richtigerweise alle Verfahren, die eine Bürgerbeteiligung vorsehen.

15 Die Motive, warum Menschen nicht an eine gottgegebene Ordnung glauben, sind so vielfältig, dass sich diese Gruppe einer Institutionalisierung entzieht. Immerhin gibt es Organisationen, wie etwa den Internationalen Bund der Konfessionslosen und Atheisten (IKBA), der sich für eine radikale Trennung von Staat und Kirchen ausspricht, dabei aber eben auch nur einen bestimmten Teil der „Ungläubigen“ repräsentieren kann.

16 Angesichts der Vielfalt des Islam kann auch hier kein Verband für sich beanspruchen, für alle Muslime sprechen zu können.

17 Es ist durchaus interessant, dass sowohl der Gutachter als auch die beiden Referenten und der Vorsitzende der Abteilung Öffentliches Recht Udo di Fabio bekennende Mitglieder der katholischen Kirche sind. 